\title{
Drink-driving Behavior Research Based on Artificial Neural Network
}

\author{
Lin Shang,Liechao Zhang(Correspongding Author) \\ School of Electronic and Information Engineering, Wuhan Technical College Of Communications \\ Wuhan, China \\ *shanglin@whtcc.edu.cn
}

Keywords: neural networks; drink-driving; sensor array

\begin{abstract}
Drink-driving, alone traffic police department regulation is not enough. Only taking the necessary scientific and technological means, drink-driving illegal behavior can be effectively controlled. In this paper, first, we established the detection of drink-driving behavior model of BP neural network; Then use the sensor array, collecting the cab and the driver's own state parameters; Next normalize samples from the samples library, which are used to training network; Finally acts of drink-driving simulation test, which can get the driver's extent of drinking and the result of driving behavior. So you can control the driving behavior according to drivers extent of drinking and the result of driving behavior.
\end{abstract}

\section{Introduction}

At present, every year due to driver traffic accidents caused by drink-driving are more than 300,000, caused the deaths of about 60,000 people, nearly $\$ 3.5$ billion in direct economic losses. Drink-driving has been looked as the primary cause of death accident by the World Health Organization. Because of the increasing risk of drink-driving accident, detection of drink-driving and controlling drivers drink-driving behavior is very important and some necessary measures must be implemented to protect the personal and property safety of the residents.

\section{OVERVIEW OF BP NEURAL NETWORK}

Artificial neural network was presented basis of the results of modern neural scientific research, which simulates human brain structure, with self-organizing, self-learning ability, By the training to determine the linear relationship between input and output variables, and establish a relationship of input and output model. In this paper, the neural network uses back propagation algorithm, also known as BP algorithm [1][2].

It is a one-way transmission of multilayer feed forward network composed of two learning processes which are information dissemination and forward error back propagation. The inputting layer neurons are responsible for receiving input from the outside world, and pass to the middle layer of the neurons. The middle layer is the internal information processing layer, responsible for information transformation. According to requirement of changing information capacity, the middle layer can be designed as a single hidden layer or more hidden layers. The last hidden layer is output layer to transfer information to each neuron, after further treatment, which complete a study of the forward propagation process. The output layer output the results to the outside world. When the actual output and expected output does not comply, enter the error back propagation stage. Errors from the output layer, can correct weights of layers by error gradient descent means, and layer-by-layer reverse communicate. Cycle process of information positive dissemination and back-propagation, is the continuous process of adjustment layers weights, is also a process neural network learning training. This process has been carried out to the network output error, which is reduced to an acceptable level, or pre-set the number of learning set up[3]. In this paper, the BP neural network is used to detect drink-driving behavior of drivers. 


\section{OVERALL SYSTEM DESIGN}

\subsection{System Principle.}

In general, if the driver drinking, he exhales breath containing alcohol, so in the cab we can detect the concentration of alcohol, while not only the driver's pulse should be accelerated and the heart beat faster, but also the driver sweat more than normal time. In addition, we also take into account seasonal factors, so temperature and humidity sensors can be used to amend detected parameters, for reducing false alarms.

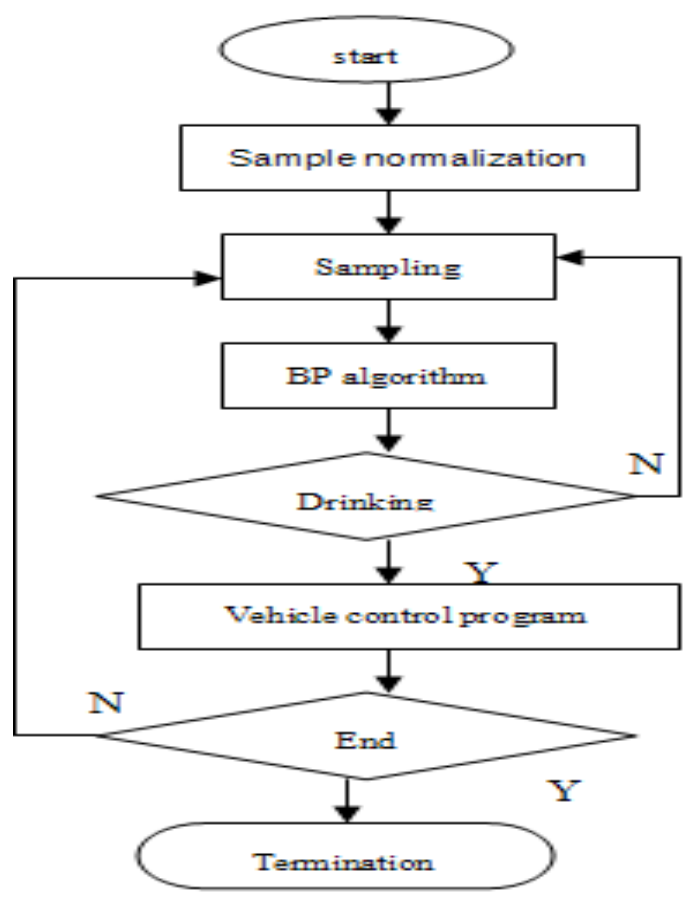

Fig. 1. System Principle chart

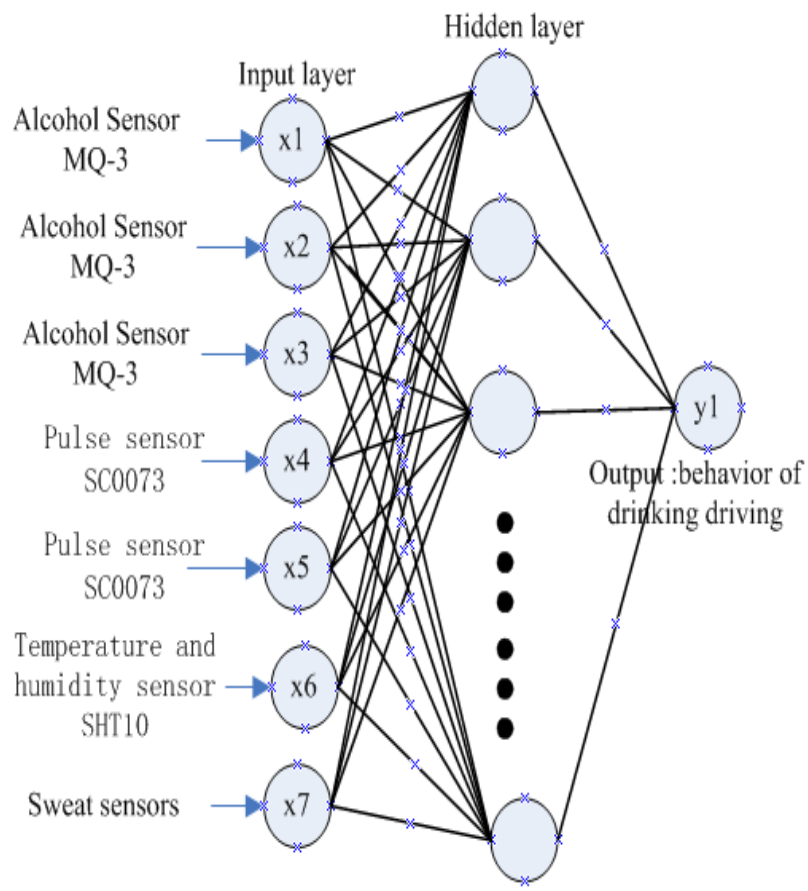

Fig. 2. BP neural network chart

In order to detect whether the driver has the drink-driving behaviors and the degree of drunkenness, you can select multiple sensors collecting the gas sample of driver cab and its status,including: three alcohol gas sensors, which installed location can effectively detect gas that drivers call out, detect the alcohol concentration mixed in cab; two pulse sensors, mainly get drivers heartbeat speed; one sweat sensor, gets drivers sweat parameter; one temperature humidity sensor, mainly detects current environment temperature and humidity, for correction other sensors detected parameter.

\subsection{Sensor selection and system flow chart.}

Alcohol concentration sensor, selecting MQ3, it has a high sensitivity, fast response and recovery, low power consumption, long life and other characteristics. Selecting SHT10 digital temperature/humidity sensor for temperature and humidity within the cab parameters, this sensor has automatic sleep function, providing an accurate measure of the relative temperature and humidity. Pulse sensor select SC0073 pulse sensor, it is a high performance, low-cost small piezoelectric pressure sensors.

When system initially operates, firstly each sensor is initialized, beginning collecting data, the collected data serve as the BP neural network input. According to the existing samples BP neural network is studied and trained, and then we can get the result that whether the drivers have drink-driving behaviors. Specifically shown in Fig. 1. 


\section{BP NEURAL NETWORK MODEL}

According to some characteristics of the driver after drinking, in this paper we choose multiple input unit, multiple hidden layer and single output, 3-layer error back propagation neural network BP used to make a comprehensive evaluation of drink-driving behavior of drivers. The driver drink-driving behavior model of BP neural network shows in Fig. 2., which X1, X2, X3, X4, X5, X6, $\mathrm{X} 7$ is input parameters, $\mathrm{Y} 1$ is the final output. Input and output parameters meaning of the input layer, see "training sample preparation and normalized" section.

\subsection{SELECTION OF PARAMETERS OF INPUT LAYER.}

In order to detect drivers of driving behavior, the BP neural network is entered 7 parameters, namely $x 1, x 2, x 3, x 4, x 5, x 6, x 7 . x 1, x 2, x 3$ are the different sampling points MQ3 sensors to get the cab alcohol concentration samples; $\mathrm{x} 4$, $\mathrm{x} 5$ are the different collection points SC0073 pulse sensors taking samples; $\mathrm{x} 6$ is the SH10 sensor to take the driving parameters of the indoor temperature and humidity; $x 7$ parameters is the acquisition of the drivers sweat. Output value is y1, said the extent of drivers drunken behavior.

\subsection{BP NEURAL NETWORK MODELING.}

\subsubsection{TRAINING SAMPLE PREPARATION AND NORMALIZED}

The initial 100 samples stored in text files, and the file stored on disk. When new samples are collected, new samples collected are put the back of the original sample file. In the samples library the first 100 samples don't change, from the 101 samples to the last samples are periodically updated; if the number of samples comes to 1500, the samples from 101 to 1500 be repeatedly updated [3][4].

Before BP algorithm runs, the first 1000 samples are selected as the sample set , and normalized to train the network.

Before making neural network, the original data must be normalized, because the data is too large to avoid the paralysis caused by the network. In the normalized process, must maintain the characteristics of the original data[5].

The method used in the normalization algorithm is as follows:

$$
X_{i}=\frac{X_{i}-\operatorname{Min}}{\operatorname{Max}-\operatorname{Min}}
$$

Max and Min are the maximum and minimum which are in the sample library.

\subsection{2 neural network structure design and parameter determination}

\section{(1)Network layers}

The existing theoretical analysis shows that hidden layers can be up to two. Only learning a discontinuous function, it requires two hidden layers; a single hidden layer neural network can map any continuous function, so we choose a one-hidden-layer BP neural network, which is three-layer neural network with a single hidden layer.

(2)hidden nodes

For BP neural network, the determination of the number of hidden neurons is the key to the success or failure. BP neural network using trial and error method to determine the number of hidden neurons, the initial hidden nodes is determined to be 3 . In repeatedly training, when the network hidden layer nodes comes to 6 , the convergence is faster, and can reach the target in a relatively short time.

In this paper the number of hidden layer is calculated through the computeHideNum function, which has two parameters, one is the number of inputting layer parameters and the other is also the number of outputting layers, the specific code as follows:

public int computeHideNum(int $\mathrm{m}$, int $\mathrm{n}$ )

\{

double $\mathrm{s}=$ Math.Sqrt $(0.43 * \mathrm{~m} * \mathrm{n}+0.12 * \mathrm{n} * \mathrm{n}+2.54 * \mathrm{~m}+0.77 * \mathrm{n}+0.35)+0.51$;

int ss $=$ Convert.ToInt $32(\mathrm{~s})$;

return $((\mathrm{s}-($ double $) \mathrm{ss})>0.5)$ ? ss $+1: \mathrm{ss}$;

\}

(3) Outputting layer nodes 
In this paper, BP neural network algorithm is a neuron output layer, that is the extent of driver drink-driving.

(4) activation function

Neuron activation function is the core, which determines the motor function of neurons. Sigmoid function is selected in the paper.

$$
f(x)=\frac{1}{1+e^{-x}}
$$

(5)neural network prediction system performance evaluation index (error sum of squares and SEE)

$$
S E E=\frac{\sum_{i}\left(y_{i}^{\prime}-y_{i}\right)^{2}}{2}
$$

System of the actual values are $(y 1, y 2 \ldots y n)$, forecast value is $\left(y^{\prime} 1, y^{\prime} 2 \ldots y^{\prime} n\right)$.

\section{CALCULATION OF THE DRIVER LEVEL DRINK DRIVING}

Trained neural network extracts the hidden nonlinear relationship in the samples, which is stored in the form of weight distribution. At running time, when the neural network is input the non-sample, it can be completed from any nonlinear mapping input space to output space, which cannot be described with mathematical regularity. Output Y1 in the paper, is the degree of driver's drink-driving behavior: If $\mathrm{Y} 1>=2$, the driver has drunken- driving behavior, if $\mathrm{Y} 1>=1$, drivers have a drinking-driving behavior, $y 1<1$, drivers are normal driving. psim is parameter of simulate function, said a sensor array at a time collecting 7 parameters. $y 1$ is the output, that is, the degree value of the driver drink-driving. public double[]Simulate(double[] psim)

\{

$$
\begin{aligned}
& \text { for (int } \mathrm{i}=0 ; \mathrm{i}<\text { inNum; } \mathrm{i}++) \\
& \mathrm{x}[\mathrm{i}]=\mathrm{psim}[\mathrm{i}] / \text { in_rate; } \\
& \text { for }(\mathrm{int} \mathrm{j}=0 ; \mathrm{j}<\text { hideNum; } \mathrm{j}++)\{ \\
& \mathrm{o} 1[\mathrm{j}]=0.0 ; \\
& \text { for }(\text { int } \mathrm{i}=0 ; \mathrm{i}<\mathrm{inNum} ; \mathrm{i}++) \\
& \mathrm{o} 1[\mathrm{j}]=\mathrm{o} 1[\mathrm{j}]+\mathrm{w}[\mathrm{i}, \mathrm{j}] * \mathrm{x}[\mathrm{i}] ; \\
& \mathrm{x} 1[\mathrm{j}]=1.0 /(1.0+\text { Math.Exp(-o1[j] - b1[j])); }
\end{aligned}
$$

for (int $\mathrm{k}=0 ; \mathrm{k}<$ outNum; $\mathrm{k}++$ )

\{

$$
\begin{aligned}
& \mathrm{o} 2[\mathrm{k}]=0.0 ; \\
& \text { for }(\mathrm{int} \mathrm{j}=0 ; \mathrm{j}<\text { hideNum; } \mathrm{j}++) \\
& \mathrm{o} 2[\mathrm{k}]=\mathrm{o} 2[\mathrm{k}]+\mathrm{v}[\mathrm{j}, \mathrm{k}] * \mathrm{x} 1[\mathrm{j}] \\
& \mathrm{y} 1[\mathrm{k}]=1.0 /(1.0+\text { Math.Exp }(-\mathrm{o} 2[\mathrm{k}]-\mathrm{b} 2[\mathrm{k}])) \\
& \mathrm{y} 1[\mathrm{k}]=\text { in_rate } * \mathrm{x} 2[\mathrm{k}] \\
& \quad \text { return } \mathrm{y} 1 ;
\end{aligned}
$$$$
\text { \} }
$$

According to the national standard of breath, alcohol content critical value less than $5 \mathrm{ug} / 100 \mathrm{ml}$ is drink-driving, greater than $50 \mathrm{ug} / 100 \mathrm{ml}$ is drunken-driving. In the testing, samples are collected by artificial way. The tester drinked and exhaled gas, we collected the samples in normal, drink-driving, drunked-driving three states. Part of the data samples used to train the network show in Fig. 3: 


\begin{tabular}{|c|c|c|c|c|c|c|c|}
\hline \multirow[b]{2}{*}{0.0018} & \multirow[b]{2}{*}{0.0012} & \multirow[b]{2}{*}{0.0009} & \multirow[b]{2}{*}{70} & \multicolumn{4}{|c|}{ Temperature } \\
\hline & & & & 71 & 0.25 & 0.455 & 0 \\
\hline 0.0021 & 0.0018 & 0.0010 & 71 & 70 & 0.25 & 0.464 & 0 \\
\hline 0.0956 & 0.0006 & 0.0067 & 78 & 77 & 0.25 & 0.561 & 1 \\
\hline 0.0067 & 0.0908 & 0.0957 & 78 & 78 & 0.26 & 0.574 & 1 \\
\hline 0. 0971 & 0. 0098 & 0. 0062 & 79 & 78 & 0.25 & 0.577 & 1 \\
\hline 0.5781 & 0.543 & 0.5343 & 82 & 81 & 0.26 & 0.745 & 2 \\
\hline 0.6543 & 0.5432 & 0.5785 & 82 & 83 & 0.25 & 0.754 & 2 \\
\hline 0.6896 & 0.5784 & 0.5986 & 83 & 84 & 0.25 & 0.764 & 2 \\
\hline
\end{tabular}

Fig. 3. Some sample

Sensor array periodically collection related parameters in the cab, as the test data; BP neural network training samples and output current value Y1. Some point, the collected sample was: $[0.6543,0.4567,0.5678,83,82,0.25,0.712]$,output $\mathrm{Y} 1=2$, shows that the driver is drunken-driving, you should take appropriate measures to control the behavior of drivers driving, so as to protect the safety of drivers and pedestrians.

\section{Conclusions}

After drinking, the driver of the stimulation of alcohol easily drive fast, coupled with the alcohol produced by fatigue, weary phenomenon and so on, thus it is difficult to maintain a high degree of driver awareness focused on the sudden emergency, unable to take effective measures to ensure traffic safety, greatly increased risk of driving.

If you use the method to install the sensor array in the cab, the cab drivers and real-time acquisition related parameters, and use artificial neural network computing the extent of drivers drink-driving behavior, then according to different drivers drink-driving behavior taking different measures to control the driver's driving behavior to reduce traffic accidents, protect the driver and the masses of life and property.

\section{Acknowledgment}

This work was supported by the key project of "12th Five-Year" planning of Education Science in Hubei province under Grant No.2013A052 and Wuhan Technical College Of Communications Scientific research and innovation team Grant No.CX2018B02 and Wuhan Technical College Of Communications Innovation and development plan of action task under Grant No. XM-15_S42-7.

\section{References}

[1]TSAI C, CHIH Y, WONG W H, et al. A hardware-efficient sigmoid function with adjustable precision for a neural network system [J]. IEEE Transactions on Circuits \&Systems: II Express Briefs, 2015, 62(11):1073-1077.

[2]Wang Deming, Wang Li, Zhang Guangming. Short-term wind speed forecast model for wind farms based on genetic BP neural network[J]. Journal of Zhejiang University: Engineering Science, 2012, 46(5): 837-841, 904.

[3]DONG Si-hui," Study on Road Traffic Accident Forecast Based on BP Neural Network", China Safty Science Journal, vol. 20, pp. 15-18, September 2010.

[4]HUAN Hong-jiang," Application of Modified BP Neural Network in Traffic Flow Forecasts", Microelectronics\&Computer,2010,1,27(1):106-110 vol. 27, pp. 106-110, January 2010.

[5]LiHong," Model Reference Adaptive Control Based On the Improved BP Neural Network", Journal of Lanzhou Jiaotong University, vol.30, pp.37-40, December 2011. 